\title{
Structure, function and evolution of the orally active insecticidal toxin complex, YenTc
}

\section{Sarah Piper, ${ }^{1,2}$, Lou Brillault ${ }^{1,2}$, Joseph Box ${ }^{1}$, Yu Shang Low ${ }^{1}$, Irene Chassagnon ${ }^{1}$, Gabriel Foley ${ }^{1}$, Nadezhda Aleksandrova ${ }^{1}$, Lauren Hartley-Tassell ${ }^{4}$, Cassandra Pegg ${ }^{1}$, Ben Schulz ${ }^{1}$, Thomas Ve$^{4}$, Shaun Lott ${ }^{3}$, Mark Hurst ${ }^{5}$, Michael Landsberg ${ }^{1,2}$}

\author{
${ }^{l}$ School of Chemistry and Molecular Bioscience, The University of Queensland, St Lucia, Australia; \\ ${ }^{2}$ Institute for Molecular Bioscience, The University of Queensland; \\ ${ }^{3}$ School of Biological Sciences, The University of Auckland; \\ ${ }^{4}$ Institute for Glycomics; \\ ${ }^{5} \mathrm{AgResearch} \mathrm{NZ}$ \\ m.landsberg@uq.edu.au
}

Yersinia entomophaga is a naturally occurring, Gram negative insect pathogen, first isolated from the diseased larvae of the New Zealand grass grub C. zealandica a decade ago [1]. Its main virulence determinant is YenTc, a heterogenous 2.4 MDa toxin complex that is a prototypical example of the $\mathrm{ABC}$ or Tc family of predominantly insecticidal toxins. YenTc is unique amongst members of this family, in that it is the only member of this class of toxins characterised to date that has a broad target host range, and which exhibits potent oral activity towards susceptible hosts without relying on a nematode symbiont for delivery. This has positioned YenTc as a potentially high value target for the development of novel biopesticides based on this class of toxins.

Previous work from our lab has yielded structures of the pore-forming A component determined by cryo-EM [2,3], the toxinencapsulating BC cage [4] of YenTc determined by X-ray crystallography, as well as the co-expressed chitinase enzymes Chil and Chi2 also determined by X-ray crysatllography [4,5], the latter of which we show are structurally incorporated into the complex. Moreover, these chitinases exhibit significant structural mobility and appear to play a role in glycan recognition at the host cell surface. Our most recent work has led to the determination (using cryo-EM) of structures for the complete YenTc holotoxin assembly in both pre-pore and pore states. Comparing these structures to those of related toxins (primarily derived from the nematode symbiont Photorhabdus luminescens), has helped us to elucidate the overall mechanism of toxin packaging, translocation and delivery. We used the knowledge derived from these structures to guide a Hidden Markov Model-based bioinformatic analysis that led to the identification of more than 800 putative toxin complexes in diverse bacterial genome sequences. Phylogenetic analysis of these putative toxins led us to conclude that $\mathrm{ABC}$ toxins cluster into 4 subtypes, and illuminate a model for the evolution of these subtypes in response to host adaptation. Finally, as part of this analysis, we identified an orphan subunit located outside the pathogenicity island of YenTc, and solved the structure of the cytotoxic effector encoded within this subunit using X-ray crystallography. This is, to our knowledege, the first cytotoxic effector associated with an $\mathrm{ABC}$ toxin to have it's structure determined.

[1] Hurst, M.R.H. et al. (2011) Int J Syst Evol Microbiol, 61(4) 844-849.

[2] Landsberg, M.J. et al. (2011) PNAS, 108(51) 20544-20549

[3] Piper, S.J. et al. (2019) Nature Commun, 10(2019) 1952.

[4] Busby, J.N. et al. (2013) Nature, 501(7468) 547-550.

[5] Busby, J.N. et al. (2011) J Mol Biol, 415(2) 359-371.

Keywords: cryoEM, bacterial toxins, phylogenetics, membrane proteins, pore-forming proteins 